\title{
Influenza Virus Subtyping by Multiplex PCR during Winter Months of 2017-2018
}

\author{
Misbah Noor1, Saif Ullah Khan Niazi1, Nadia Tayyab2, Syed Adeel Gardezi2, \\ Eijaz Ghani ${ }^{1}$ and Muhammad Tahir Khadim ${ }^{3}$
}

\begin{abstract}
Objective: To determine the frequency of infections caused by Influenza viruses, i.e. Influenza A (H1N1) pdm09, Influenza A $(\mathrm{H} 3 \mathrm{~N} 2)$ and Influenza B in patients presenting with respiratory tract infections, i.e. influenza-like illness (ILI) and severe acute respiratory illness (SARI).

Study Design: Descriptive, cross-sectional study.

Place and Duration of Study: Department of Virology, Armed Forces Institute of Pathology (AFIP), Rawalpindi, from October 2017 to February 2018.

Methodology: A total of 624 samples from patients with respiratory tract infections (both ILI and SARI) were included in the study. Specimens collected from the patients included nasal swabs and throat swabs, which were transported in viral transport medium (VTM) to Virology Department, AFIP. Multiplex PCR was done for Influenza A (H1N1) pdm09, Influenza A (H3N2) and Influenza B.

Results: A total of $200(32 \%)$ samples were found to be positive for Influenza viruses. Out of total 624 samples analysed, $220(35.3 \%)$ were from females and $404(64.7 \%)$ from males. Among these, $510(81.7 \%)$ presented with ILI and $114(18.3 \%)$ with SARI. Among positive samples, $120(19.2 \%)$ samples were positive for H1N1, 61 (9.8\%) for H3N2 and $19(3 \%)$ were positive for Influenza B. Highest number of positive cases occurred in the month of January, i.e. $148(74 \%)$ cases. Only 3 (2.5\%) patients out of 120 infected with H1N1 were in age group-I (0-5 years). While in age group-II (6-30 years), age group-III (31-60 years), and age group-IV (>60 years); $39(32.5 \%), 63(52.5 \%)$ and $15(12.5 \%)$ patients were infected by $\mathrm{H} 1 \mathrm{~N} 1$, respectively. Maximum patients with H3N2 infection were in age group-III; 30 (49.2\%) of the total 61 . Commonest Influenza subtype in age group-IV was H3N2 found in 20 (32.8\%) patients, followed by H1N1 in 15 (12.5\%) patients.

Conclusion: The dominant subtype in our set-up, during winter of 2017-2018, was Influenza A (H1N1) pdm09. Highest numbers of positive cases were recorded in the month of January. People with ILI and SARI should be tested for Influenza viruses to avoid unnecessary use of antibiotics.
\end{abstract}

Key Words: Influenza A (H1N1) pdm09, Influenza A (H3N2), Influenza B, Influenza-like illness (ILI), Severe acute respiratory illness (SARI).

\section{INTRODUCTION}

Influenza viruses are rapidly evolving pathogens that cause respiratory tract infections with significant morbidity and mortality among humans. ${ }^{1}$ These are notorious for genetic variability that results from antigenic drift and antigenic shift.2,3 Unlike, seasonal influenza epidemics that result from point mutations, the pandemics result from genetic re-assortment resulting in novel viruses to which human population has no immunity. These pandemics cause severe illness and death as compared to seasonal influenza. Five famous pandemics of Influenza A have been reported over the past 100 years. ${ }^{4}$ The 2009 pandemic resulted in over 209,000 laboratory confirmed cases of influenza and over 3205 deaths in USA alone. 5

Department of Virology1 / Microbiology2 / Pathology33, Armed Forces Institute of Pathology (AFIP), Rawalpindi, Pakistan

Correspondence: Dr. Misbah Noor, Department of Virology, Armed Forces Institute of Pathology (AFIP), Rawalpindi,

Pakistan

E-mail: misbahnoor001@gmail.com

Received: June 21, 2018; Accepted: November 30, 2018
Influenza-like illness (ILI) carries a significant economic burden in the form of reduced productivity and high healthcare costs. ${ }^{6}$ Complications are usually reported in children $<2$ years, elderly $>65$ years, pregnant women and those with chronic medical conditions. In 2009 pandemic, $>90 \%$ deaths occurred in old age group. ${ }^{7}$ In temperate regions, annual winter epidemics occur; whereas in tropical regions, sporadic outbreaks occur throughout the year. 8,9

We conducted this study to determine the frequency of infections caused by influenza viruses in patients presenting with ILI and SARI. ${ }^{10}$ This would help us in making informed decisions to avoid unnecessary use of antibiotics and prescribing yearly vaccination. It will also help in documenting the influenza virus subtypes and provide data for future comparison, if the circulating subtypes change in this region.

\section{METHODOLOGY}

It was a descriptive, cross-sectional study of five months duration (October 2017 to February 2018). Patients with the clinical diagnosis of ILI and SARI of all age groups and both genders were included in the study. Patients 
with chronic respiratory conditions, such as asthma, and chronic obstructive pulmonary disease (COPD) and those with lower respiratory ailments were excluded from study. Nasal and throat swabs were collected and transported to Virology Department, Armed Forces Institute of Pathology (AFIP), Rawalpindi, in viral transport medium (VTM). Those samples were further transported to National Institute of Health $(\mathrm{NIH})$, Islamabad, at optimum temperature $\left(2-6^{\circ} \mathrm{C}\right)$. Multiplex RT-PCR was done for subtyping of influenza viruses, using WHO recom-mended Influenza Real-Time RTPCR panel. Specific primers targeting conserved regions of hemagglutinin-neuraminidase $(\mathrm{HN})$ genes were used for subtyping of influenza viruses.

Data was entered in SPSS version 21 and mean \pm SD were used for quantitative variables and qualitative variables were expressed by frequency along with percentages. Chi-square test was used to determine association between variables like gender, disease group, month, influenza virus subtype and age groups. A $p$-value of $<0.05$ was considered as statistically significant.

\section{RESULTS}

A total of 624 samples of patients with ILI/SARI were tested during the study period. Real time PCR test revealed that out of 624 samples, 200 (32\%) were positive for human influenza viruses. Out of all samples analysed, 220 (35.3\%) were from females and 404 $(64.7 \%)$ from males as shown in Figure 1.

Among positive cases, the distribution of influenza viruses showed $\mathrm{H} 1 \mathrm{~N} 1$ positivity in $120(19.2 \%), \mathrm{H} 3 \mathrm{~N} 2$ in
$61(9.8 \%)$, and influenza B in $19(3 \%)$ cases. Positivity of influenza viruses was significantly higher in ILI group $167 / 624(26.7 \%)$ than in the SARI group, 33/624 (5.2\%) as shown in Table I. All patients with Influenza B positivity presented with ILI and there was no case of SARI due to Influenza B.

The highest number of positive cases occurred in the month of January, i.e. 148 (74\%) out of 200 positive cases among the 394 total tested during this month. Chisquare test was applied to test the association of these two variables, i.e. month and positive result and $p$-value was 0.002 , which shows that there is a correlation between influenza positivity and the month of year as shown in Table II.

Chi-square test was also applied between the variables of influenza virus positivity and different age groups. $P$-value was 0.037 , which also shows significant association of virus positivity with age groups as shown in Table III.

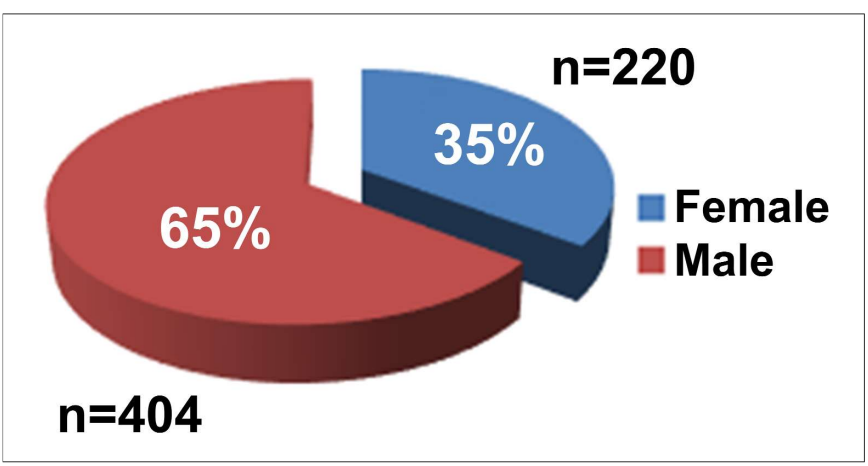

Figure 1: Gender distribution.

Table I: Distribution of positive virus infections according to disease groups.

\begin{tabular}{l|cccc|c}
\hline \multirow{2}{*}{ Disease group } & \multicolumn{4}{|c|}{ Influenza subtypes } & \multirow{2}{*}{ Total } \\
\cline { 2 - 6 } & Negative & H1N1 & H3N2 & INF B & \\
\hline ILI & $343(67.2 \%)$ & $102(20 \%)$ & $46(9.1 \%)$ & $19(3.7 \%)$ & $510(81.7 \%)$ \\
\hline SARI & $81(71.05 \%)$ & $18(15.78 \%)$ & $15(13.15 \%)$ & 0 & $114(18.3 \%)$ \\
\hline Total & $424(67.94 \%)$ & $120(19.23 \%)$ & $61(9.77 \%)$ & $19(3.04 \%)$ & $624(100 \%)$ \\
\hline
\end{tabular}

Table II: Monthly distribution of positive cases.

\begin{tabular}{|c|c|c|c|c|c|}
\hline \multirow[t]{2}{*}{ Month } & \multicolumn{4}{|c|}{ Influenza virus subtypes } & \multirow[t]{2}{*}{$\mathrm{p}$-value } \\
\hline & Negative & $\mathrm{H} 1 \mathrm{~N} 1$ & $\mathrm{H} 3 \mathrm{~N} 2$ & INF B & \\
\hline Oct & $6(1.4 \%)$ & $1(0.8 \%)$ & 0 & 0 & 0.002 \\
\hline Nov & $10(2.4 \%)$ & 0 & 0 & 0 & \\
\hline Dec & $8(1.9 \%)$ & $2(1.7 \%)$ & 0 & 0 & \\
\hline Jan & $246(62.4 \%)$ & $95(24.1 \%)$ & $45(11.4 \%)$ & $8(2 \%)$ & \\
\hline Feb & $154(36.3 \%)$ & $22(18.3 \%)$ & $16(26.2 \%)$ & 11 (57.9\%) & \\
\hline
\end{tabular}

Table III: Distribution of virus positive cases according to age group.

\begin{tabular}{l|cccc|c}
\hline Age groups & Negative & H1N1 & H3N2 & INF B & p-value \\
\hline $0-5$ years $(\mathrm{gp}-\mathrm{I})$ & $11(2.6 \%)$ & $3(2.5 \%)$ & 0 & 0 & 0.037 \\
6-30 years $(\mathrm{gp}-\mathrm{II})$ & $115(27.1 \%)$ & $39(32.5 \%)$ & $11(18 \%)$ & $6(31.6 \%)$ & \\
$31-60$ years $(\mathrm{gp}-\mathrm{III})$ & $189(44.6 \%)$ & $63(52.5 \%)$ & $30(49.2 \%)$ & $6(31.6 \%)$ & \\
$>60$ years (gp-IV) & $109(25.7 \%)$ & $15(12.5 \%)$ & $20(32.8 \%)$ & $7(36.8 \%)$ & \\
\hline Total & 424 & 120 & 61 & 19 & \\
\hline
\end{tabular}


Chi-square test was also applied between influenza virus positivity and gender. This was not statistically significant with a $p$-value of 0.230 .

\section{DISCUSSION}

This study demonstrates the activity of influenza virus infection during winter months of 2017-2018 in Northern Pakistan. The overall prevalent strain in our study was Influenza A (H1N1) pdm09, followed by Influenza A (H3N2). According to WHO, around the globe, Influenza A positivity was $45 \%$ and Influenza B positivity was $55 \%$. Low influenza activity was reported from Southern Asia and South East Asia. Worldwide, Influenza A (H1N1) positivity was $60.4 \%$ and Influenza A (H3N2) was $39.6 \% .11$

In a regional study by Dangi et al., ILI due to influenza viruses was reported more than SARI. ${ }^{12}$ Similar trend was observed in this study. In contrast to this study, the previous study reported more influenza $B$ positivity as compared to influenza A. ${ }^{12}$ According to recent CDC surveillance report, Influenza A positivity in USA this year was $78.3 \%$ and Influenza B positivity was $21.7 \%$. The dominant influenza subtype from USA was influenza A (H3N2), which was different from results of our study as our dominant subtype continues to be influenza $A$ (H1N1). ${ }^{13}$ H3N2 was associated with severe influenza disease and this strain dominated during 3 of the last 5 severe influenza seasons. ${ }^{14}$

A large multicentric cohort study conducted during 2009 to 2015 showed that influenza A cases outnumbered influenza $B$ with more hospital admissions and complications reported in influenza $A(H 1 N 1)$ positive cases than influenza A (H3N2). ${ }^{15}$

Khalid et al. reported 10\% Influenza A and 3\% Influenza B incidence in children with lower respiratory tract disease. 16 In this study, only $1.5 \%$ Influenza A (H1N1) infection was reported in children $<5$ years of age. Influenza mortality increases drastically with age, the risk of death was 11 -fold higher in people aged 80 years than in those 66-69 years. ${ }^{17}$ In this study, in patients $>60$ years, Influenza A and Influenza B positivity was $25 \%$ and $3.5 \%$, respectively with more cases of Influenza A $(\mathrm{H} 3 \mathrm{~N} 2)$ than Influenza A (H1N1).

Although most cases of influenza will recover without sequalae, influenza can cause serious illness and death, particularly among older adults, very young children, pregnant women, and those with certain chronic medical conditions. CDC's Advisory Committee on Immunization Practices (ACIP) recommends annual immunization in these groups to reduce morbidity and mortality. ${ }^{18}$ No such recommendations regarding vaccination exist in Pakistan, so the vaccine coverage is very low. Moreover, starting antivirals as soon as possible, especially within 48 hours of onset of symptoms, will help reduce disease severity and loss of function. ${ }^{19}$

\section{CONCLUSION}

Influenza viruses are an important cause of ILI and SARI. The dominant subtype of influenza viruses in Northern Pakistan during 2017-2018 winter months was Influenza A (H1N1), followed by Influenza A (H3N2). Timely collection of specimen and its testing can result in prompt and appropriate diagnosis and can help avoid unnecessary use of antibiotics. This study will also help in providing data for future comparisons about circulating influenza subtypes in Northern Pakistan.

\section{REFERENCES}

1. Klingen TR, Reimering S, Loers J, Mooren K, Klawonn F, Krey T, et al. Sweep dynamics plots: Computational identification of selective sweeps to monitor the adaptation of influenza $A$ viruses. Sci Rep 2018; 8:373

2. Bonin E, Queguiner S, Wondstra C, Gorin S, Barbier N, Harder TC, et al. Molecular subtyping of European swine influenza viruses and scaling to high throughput analysis. Virol $J$ 2018; 15:7

3. Webster RG, Govorkova EA. Continuing challenges in influenza. Ann N Y Acad Sci 2014; 1323:115-39.

4. Gagnon A, Acosta E, Hallman S, Bourbeau R, Dillon LY, Ouellette $\mathrm{N}$, et al. Pandemic paradox: Early life H2N2 pandemic influenza infection enhanced susceptibility to death during the 2009 H1N1 Pandemic. MBio 2018; 9:e02091-17

5. Presanis AM, De Angelis D, Hagy A, Reed C, Riley S, Cooper BS, et al. The severity of pandemic H1N1 influenza in the United States, from April to July 2009: A Bayesian Analysis. PLoS Med 2009; 6:e1000207.

6. Adiga A, Chu S, Eubark S, Kuhlman C, Lewis B, Marathe A, et al. Disparities in spread and control of influenza in slums of Delhi: finding from an agent-based modeling study. BMJ Open 2018; 8:e017353.

7. Jain S, Kamimoto L, Bramley AM, Schmitz AM, Benoit SR, Louie J, et al. Hospitalized patients with 2009 H1N1 Influenza in the United States, April-June 2009. N Engl J Med 2009; 361: 1935-44

8. Geoghegan JL, Saavedra AF, Duchêne S, Sullivan S, Barr I, Holmes EC. Continental synchronicity of human influenza virus epidemics despite climactic variation. PLoS Pathog 2018; 14:e1006780.

9. Steel J, Palse P, Lowen AC. Transmission of a 2009 pandemic influenza virus shows a sensitivity to temperature and humidity similar to that of an H3N2 seasonal strain. J Virol 2011; 85: 1400-2

10. Human infection with pandemic (H1N1) 2009 virus: Updated interim WHO guidance on global surveillance. WHO 2009.

11. Influenza Update $\mathrm{N}^{\circ} 311$. WHO, 2018, www.who.int/ influenza/ surveillance_monitoring/updates/2018_03_19_surveillance_u pdate_311

12. Dangi T, Jain B, Singh AK, Mohan M, Dwivedi M, Singh JV, et al. Influenza virus genotypes circulating in and around Lucknow, Uttar Pradesh, India, during post pandemic period, August 2010 - September 2012. Indian J Med Res 2014; 139: 418-26

13. https://www.cdc.gov/flu/weekly/index.htm

14. Allen JD, Ross TM. H3N2 influenza viruses in humans: Viral mechanisms, evolution, and evaluation. Hum Vaccin Immunother 2018; 14:1840-7. 
15. Sam JI. The burden of human influenza in Malaysia. Med $J$ Malaysia 2015; 70:127-30

16. Khalid S, Ghani E, Ayyub M. Study on etiology of viral lower respiratory tract infections in children under 10 years of age. J Virol Antivir Res 2016; 5:1-3

17. Bahadoran A, Lee SH, Wang SM, Manikam R, Rajarajeswaran J, Raju CS, et al. Immune responses to influenza virus and its correlation to age and inherited factors. Front Microbiol 2016; 7:1841.
18. Grohskopf LA, Sokolow LZ, Broder KR, Walter EB, Bresee JS, Fry AM, et al. Prevention and control of seasonal influenza with vaccines: Recommendations of the advisory committee on immunization practices - United States, 2017-18 influenza season. MMWR Recomm Rep 2017; 66:1-20.

19. Torner N, Martinez A, Basile L, Mosquera M, Anton A, Rius C, et al. Descriptive study of severe hospitalized cases of laboratory confirmed influenza during five epidemic seasons (2010-2015). BMC Res Notes 2018; 11:244.

$\cdots \cdot \hat{\imath} \cdot \ldots$ 